\title{
EDITORIAL
}

\section{TRENDS ON CEREBROSPINAL FLUID INVESTIGATION: AN OVERWIEW*}

The study of cerebrospinal fluid (CSF) is an old tradition in São Paulo. From 1930, Lange and later Reis have undertaken such studies. Later this tradition was kept alive by their collaborators. Lange's coworkers continued the study of different clinical and scientific problems, and in due time have become the centre per se, at top level, specialised in CSF in neurological and neurotropical diseases in South America.

In West Europe, in 1950, three laboratories were concerned with the clinical biochemistry of the CSF: J.N. Cumings' laboratory in London; H. Bauer's in Hamburg and ours in Antwerp, under the leadership of D. Karcher and myself.

At the same time, in East Europe, extensive studies have been pursued concerned with the cytology of the CSF. Sayk was then a forerunner. Cytological studies of the CSF sediments have helped establish the diagnosis of primary tumours in the central nervous system (CNS), studying the astroprotein, the glial cells present on examination in the CSF, and in general, also secondary tumours. Haematological diseases, in particular leucaemia, were detected using the same type of examinations. At the time, biochemical as well as cytological studies of the CSF were seldom performed in the United States.

Soon after 1950, more precise activities have evolved. In 1957, a symposium on the CSF was held, at the international neurological congress in Brussels. When the neurochemistry research group of the World Federation of Neurology (WFN) was founded, from 1959 on, part of its activities were dedicated to the CSF. Later still, a new research group devoted to the CSF started, and is presently under the leadership of Prof. Christian Sindic.

In addition reports on the activities and the progresses made were published. The books of Schmidt on the CSF were edited and in 1994 Knight reviewed recent publications.

New techniques were made available. Among them, the electrophoresis of the CSF proteins has been most successfully applied, permitting to confirm numerous diagnoses, and in particular demonstrating the IgG fractionation in multiple sclerosis. Immunological studies applied to the CSF were less successful. More recently the use of PCR helped detect and identify proteins or protein fragments and viruses in the CSF. During a symposium of the WFN Research Group on CSF in Amsterdam, this was brilliantly demonstrated.

Many questions remain unsolved regarding the biochemistry of the CSF. Among others, how classify the obtained results: according to their neurological or their biochemical characteristics? Other difficulties arise due to the fact that the number of CSF samples and their volume are limited. The development of CSF research remains slow as methods applied to the blood in clinical biology, were applied to to the CSF. They never were sufficiently specific.

This explains why the evolution of CSF research was slow to develop and that the methods usually used like enzyme, amino-acid and transmitters dosages were almost abandoned.

The following can summarise some recent results concerning the CSF biochemistry:

1 - in different protein studies using PCR, the CSF helped identify infection. The CSF protein electrophoresis revealed the first human biological anomaly in multiple sclerosis patients, but also in other diseases. Recent publications report in particular immunological anomalies in cases of amyotrophic lateral sclerosis. In chronic poliomyelitis, an immunological reaction is observed. In heredo-ataxia, a genetic approach has been applied. It has not led to satisfactory conclusions, contrarily to those obtained in other neurological diseases. Polyneuritis has been subdivided, after CSF examination, into different categories. 
2- not to forget, the studies in HIV: the most recent have shown how to follow the treatment applied to the disease with RNA determinations in the CSF.

3 - the study of CSF has helped gather information concerning organic dementia. In spongiform encephalopathies gamma-gamma enolase determinations help confirm the diagnosis. Until now these determinations have been applied only to human CSF. Would it be of interest to carry them out in animals? In dementia of Alzheimer type (DAT) several substances in the CSF have been studied. As a whole they do not seem to bring any interesting information referring to the study of acetylcholine metabolism. The study of other transmitters, NO for instance, which is shown to be toxic, has brought some indications. The study of the tau protein seems most promising: it is increased in the CSF of DAT. The increase of the tau protein helps confirm the diagnosis of DAT and is a good procedure establish the to establish the diagnosis. Protein research gives some indications in organic dementias and promises to be most effective for future investigations.

4- new approaches have drawn much attention as for instance, the differential diagnosis between Rett syndrome and autism studying beta endorphin and glutamate. Modifications of the CSF aminoacids are observed in children affected with renal diseases, as reported quite recently.

To cover all that has been done during the past years on CSF, numerous publications need to be consulted. They concern principally multiple sclerosis and organic dementias.

\section{ARMAND LOWENTHAL**}

* Presented at the Cerebrospinal Fluid Symposium of the XVIII Congresso Brasileiro de Neurologia (São Paulo, 1998).

** Founding Member of the Research Group of Cerebrospinal Fluid of the World Federation of Neurology (Antwerp, Belgium. FAX 323237 4130). 